\title{
Modified Bacterial Foraging Optimization for Fuzzy Mean-Semivariance-Skewness Portfolio Selection
}

\author{
Xinzheng $\mathrm{Wu}^{1}$, Aiqing $\mathrm{Gao}^{2(\otimes)}$, and Xin Huang ${ }^{2}$ \\ ${ }^{1}$ College of Economics, Shenzhen University, Shenzhen, China \\ 2 College of Management, Shenzhen University, Shenzhen, China \\ gaoaiqing_alina@163.com
}

\begin{abstract}
In this paper, a novel bacterial foraging optimization with decreasing chemotaxis step combined with sine function is employed to solve a fuzzy portfolio optimization with a modified mean-semivariance-skewness model which includes the transaction fee and no short sales. First of all, a decreasing chemotaxis step combined with sine function (BFO-SDC) takes the place of constant chemotaxis step size. It is a nonlinear decreasing strategy at every iteration of the algorithm. And then, the variance is replaced by semivariance and skewness is taken into account in order to generate asymmetry of return distributions to overcome the inadequacy of the standard mean-variance model. Finally, fuzzy variables are used to express the uncertain and imprecise elements in the decision-making process. The results of the simulation show that the model can be solved more reasonably and effectively by BFO-SDC than the original bacterial foraging optimization.
\end{abstract}

Keywords: Bacterial foraging optimization $\cdot$ Sine function $\cdot$ Fuzzy portfolio optimization $\cdot$ Mean-semivariance-skewness

\section{Introduction}

The mean-variance model (MVM) originally formulated by Markowitz [1] plays a critical and vital role in modern finance theory. Inspired by the Markowitz's idea, numerous extensions have been proposed, e.g., multi-period mean-variance formulation [2], behavioral mean-variance portfolio selection [3]. Nevertheless, one of the assumptions of MVM is that the returns of assets are symmetrically distributed. Actually, in reality, there are shreds of empirical evidence $[4,5]$ suggesting that many security returns follow the asymmetrical distribution. People start to considerate asymmetry of return distributions to overcome the inadequacy of the MVM. The one is that Ibbotson [6] and Prakash et al. [7] show that it can generate a higher return when skewness is considered in the decision-making process. As a result, some scholars such as Prakash et al. [7] extend MVM to mean-variance-skewness model (MVSM). The other directly use downside risk measure to avoid sacrificing a large amount of expected return in removing both high and low return extremes. Semivariance [8] which is clear, comparatively simple 
and direct in mirroring investors' intuition about risk is one of the well-known downside risk measures.

Moreover, another basic assumption of MVM is that the asset markets in the future can accurately reflect the asset data in the past. However, it is impossible to produce correct expectations for future risks and returns based on historical data exclusively in the uncertain economic environment. Thus, the fuzzy set theory [9] is well suited to deal with vagueness and uncertainty of any stock market attributes in portfolio optimization (PO).

As a fresh comer of swarm intelligence (SI), bacterial foraging optimization (BFO) [10] has gotten much attention from different areas, and widely used in many realworld problems including supply chain optimization problem [11], flexible job-shop scheduling problem [12], and vehicle routing problem [13]. And a modified BFO with linear decreasing chemotaxis step (BFO-LDC) has been already successfully applied to solve the PO [14].

Sine function, one of the trigonometric functions, has been applied in the generation of n-scroll attractors [15], the design of ship course-keeping autopilot [16], sound encryption scheme [17], etc.

Based on the analysis of the recent literature, the problems about how to modify MVM to become more suitable for the real stock market and promote the ability of BFO to achieve better solutions in the PO attracts our attention. In this paper, we propose a novel BFO with decreasing chemotaxis step combined with sine function (BFO-SDC) and build a more complex PO model. The main contributions of this paper are listed as follows. First of all, a decreasing strategy based on sine function replaces the constant chemotaxis step size to investigate the optimization ability of BFO through combing decreasing strategy with sine function to create a nonlinear decreasing strategy. Next, use semivariance to replace variance and take skewness into account to generate asymmetry of asset distributions. And then, transaction fees, no short sales are also included. Finally, fuzzy logic [18] is used to express uncertainty to make it suitable for representing the intrinsic vagueness nature of the PO problem.

The remainder of the paper is organized as follows. Section 2 provides some introductory definitions and concepts. Section 3 reviews BFO and describes BFO-SDC. Section 4 gives a description of the modified fuzzy mean-semivariance-skewness model and related computational steps. The experimental results and analyses are shown and discussed in Sect. 5. In the end, concluding remarks are given in Sect. 6.

\section{Preliminaries}

The theories related to the definitions needed in the rest of the paper are briefly introduced in this section. The assistance of a membership function $\mu_{\mathrm{A}}(x) \rightarrow[0,1]$, which is a single value between zero and one represents the degree of membership of the various elements.

Definition 1 [19]. A trapezoidal fuzzy number $A$ with tolerance interval $[a, b]$, leftwidth $\alpha>0$ and right-width $\beta>0$ is fully determined by the quadruplet $(a, b, \alpha, \beta)$. 
And its membership function is given by

$$
\mu_{A}(X)= \begin{cases}1-\frac{a-X}{\alpha}, & \text { if } a-\alpha \leq X \leq a \\ 1, & \text { if } a \leq X \leq b \\ 1-\frac{X-b}{\beta}, & \text { if } b \leq X \leq b+\beta \\ 0, & \text { if otherwise }\end{cases}
$$

Definition 2 [20]. A fuzzy number $A$ with the $\gamma$-level set is expressed as $[A]^{\gamma}=\left[\alpha_{1}(\gamma)\right.$, $\alpha_{2}(\gamma)$ ] for all $\gamma>0$ and the $\gamma$-level set of $A$ can be computed as

$$
[A]^{\gamma}=[a-(1-\gamma) \alpha, b+(1-\gamma) \beta](\gamma \in[0,1])
$$

The possibilistic mean value of $A$ is given by the following.

$$
E(A)=\int_{0}^{1} \gamma\left[\alpha_{1}(\gamma)+\alpha_{2}(\gamma)\right] d \gamma=\frac{a+b}{2}+\frac{\beta-\alpha}{6}
$$

Definition 3 [21]. The upper and lower semivariances of $A$ are defined as

$$
\begin{aligned}
& \operatorname{Var}^{+}(A)=\int_{0}^{1} \gamma\left[E(A)-\alpha_{2}(\gamma)\right]^{2} d \gamma=\left(\frac{b-a}{2}+\frac{\beta+\alpha}{6}\right)^{2}+\frac{\beta^{2}}{18} \\
& \operatorname{Var}^{-}(A)=\int_{0}^{1} \gamma\left[E(A)-\alpha_{2}(\gamma)\right]^{2} d \gamma=\left(\frac{b-a}{2}+\frac{\beta+\alpha}{6}\right)^{2}+\frac{\alpha^{2}}{18}
\end{aligned}
$$

Definition 4 [22]. For any two given fuzzy numbers $A$ with $[A]^{\gamma}=\left[\alpha_{1}(\gamma), \alpha_{2}(\gamma)\right]$ and $B$ with $[B]^{\gamma}=\left[b_{1}(\gamma), b_{2}(\gamma)\right]$ for all $\gamma \in[0,1]$, the upper and lower possibilistic semicovariance between $A$ and $B$ are given as follows.

$$
\begin{aligned}
& \operatorname{Cov}^{+}(A, B)=2 \int_{0}^{1} \gamma\left[E(A)-\alpha_{2}(\gamma)\right] \cdot\left[E(B)-b_{2}(\gamma)\right] d \gamma \\
& \operatorname{Cov}^{-}(A, B)=2 \int_{0}^{1} \gamma\left[E(A)-\alpha_{1}(\gamma)\right] \cdot\left[E(B)-b_{1}(\gamma)\right] d \gamma
\end{aligned}
$$

Especially, when $A$ and $B$ are symmetric fuzzy numbers, $\operatorname{Var}^{+}(A)=\operatorname{Var}^{-}(A), \operatorname{Var}^{+}(B)$ $=\operatorname{Var}^{-}(B)$ and $\operatorname{Cov}^{+}(A, B)=\operatorname{Cov}^{-}(A, B)$.

Lemma 1 [20]. Let $A$ and $B$ be two fuzzy numbers. For any real numbers $\mu$ and $\lambda$, the following conclusion can be drawn.

$$
E(\lambda A \pm u B)=\lambda E(A) \pm u E(B)
$$

Theorem 1 [23]. Let $A_{1}, A_{2} \ldots A_{n}$ be $\mathrm{n}$ fuzzy numbers, and let $\lambda_{1}, \lambda_{2}, \ldots \lambda_{n}$ be $\mathrm{n}$ real numbers. Then

$$
\begin{gathered}
\operatorname{Var}^{+}\left(\sum_{i=1}^{n} \lambda_{i} A_{i}\right)=\sum_{i=1}^{n} \lambda_{i}^{2} \operatorname{Var}^{+}\left(A_{i}\right)+2 \sum_{i<j=1}^{n} \lambda_{i} \lambda_{j} \operatorname{Cov}^{+}\left(A_{i}, A_{j}\right) \\
\operatorname{Var}^{-}\left(\sum_{i=1}^{n} \lambda_{i} A_{i}\right)=\sum_{i=1}^{n} \lambda_{i}^{2} \operatorname{Var}^{-}\left(A_{i}\right)+2 \sum_{i<j=1}^{n} \lambda_{i} \lambda_{j} \operatorname{Cov}^{-}\left(A_{i}, A_{j}\right)
\end{gathered}
$$

Definition 4 [24]. Let $A$ be a fuzzy variable with finite expected value $e$. Then its skewness is defined as

$$
S k(A)=E\left[(A-e)^{3}\right]
$$




\section{BFO and BFO-SDC}

\subsection{Bacterial Foraging Optimization}

Inspired by the social foraging behavior of the E. coli bacteria, BFO is an evolutionary optimization technique that has been applied to multitudinous optimization problems. Based on Liu and Passino's [10] discussion of how the control system of E. coli instructs and forages, bacteria migrate to nutrient-rich areas through the flagella. The cycle of optimization is divided into four steps: chemotaxis, swarming, reproduction, and elimination and dispersal. A brief introduction is as follows.

\subsubsection{Chemotaxis}

Bacteria "swim" or "tumble" via flagella. By switching between the two modes of motion, bacteria move randomly. Supposed $\theta^{i}(j, k, l)$ indicates the information of the $i$ th bacterium including $j$ th chemotaxis, $k$ th reproduction, and $l$ th elimination and dispersal step. $C$ is the step size taken by swimming in a random direction. $\Delta$ represents the vector whose element is in the random direction of $[-1,1]$. When calculating chemotaxis, the mathematical expression of bacterial movement is

$$
\theta^{\mathrm{i}}(\mathrm{j}+1, \mathrm{k}, \mathrm{l})=\theta^{\mathrm{i}}(\mathrm{j}, \mathrm{k}, \mathrm{l})+\mathrm{C}(\mathrm{i}) \frac{\Delta(\mathrm{i})}{\sqrt{\Delta^{\mathrm{T}}(\mathrm{i}) \Delta(\mathrm{i})}}
$$

\subsubsection{Swarming}

Researchers have found that E. coli cells use complex communication mechanisms such as quorum-sensing, chemotactic signaling, and plasmid exchange to form highly structured colonies to improve environmental fitness. Swarming of bacteria can be represented by

$$
\begin{aligned}
J_{c c}(\theta, P(j, k, l)) & =\sum_{i=1}^{n} J_{c c}^{i}\left(\theta, \theta^{i}(j, k, l)\right)+\sum_{i=1}^{n}\left[h_{\text {repelent }} \exp \left(-w_{\text {repelent }} \sum_{m=1}^{p}\left(\theta_{m}-\theta_{m}^{i}\right)^{2}\right)\right] \\
& +\sum_{i=1}^{n}\left[-d_{\text {attract }} \exp \left(w_{\text {attract }} \sum_{m=1}^{p}\left(\theta_{m}-\theta_{m}^{i}\right)^{2}\right)\right]
\end{aligned}
$$

where $J_{c c}(\theta, P(j, k, l))$ is an objective function that changes with the population distribution state, reflecting the trend of the relative distance between bacteria and the optimal individual. $S$ represents the population size, and $P$ indicates the dimension of the optimization problem. $d_{\text {attract }}, w_{\text {attract }}, h_{\text {replent }}$ and $w_{\text {replent }}$ are different coefficients chosen properly. 


\subsubsection{Reproduction}

The bacteria are ranked by their health, with half of the less healthy bacteria dying and each of the healthier half splitting into two, leaving the total number of bacteria unchanged.

\subsubsection{Elimination and Dispersal}

It is well known that the life of a bacterial population can change gradually. All bacteria in an area may be killed, or a colony may disperse and emerge in a new environment. Although this change can disrupt the chemotaxis process, it is beneficial for bacteria to jump out of the local optimal domain and improve the global search ability. In this process, some bacteria die by a given probability $P_{e d}$ and then reappear randomly throughout the search space.

\subsection{BFO with Decreasing Chemotaxis Step Based on Sine Function}

This part mainly introduces the application of the decreasing chemotaxis step based on sine function in algorithm improvement.

\subsubsection{Sine Function in Chemotaxis Step}

Sine function is a trigonometric function that is a kind basic theory of trigonometry. And the function value changes nonlinearly.

In our proposed method, a decreasing chemotaxis step length combined with sine function is used over iterations. It starts with a large value and decreases to a small value at the maximal number of iterations. The mathematical representation of the BFO method is given as

$$
C(i)=C_{\max }-\left(C_{\max }-C_{\min }\right) \cdot \sin \left(\frac{\pi}{2} \cdot \frac{j}{N_{e d} \cdot N_{r e} \cdot N_{c}}\right)
$$

In the formula, $N_{c}$ denotes the number of chemotactic. $N_{e d}$ is the number of elimination-dispersal. $N_{r e}$ represents the number of reproduction. $C_{\min }$ and $C_{\max }$ are real numbers and $j$ is the current number of iterations.

\section{Bacterial Foraging Optimization for Portfolio Selection Problems}

\subsection{Portfolio Selection Model}

In this section, a mean-semivariance-skewness model that considers no short sales and the transaction fee in portfolio optimization is explained. The model can be described as follows.

$$
\begin{aligned}
\operatorname{Min} F(X) & =\min \{\lambda g(X)-(1-\lambda)[f(X)+h(X)]\} \\
\text { s.t. } & \left\{\begin{array}{l}
\sum_{i=1}^{d} X_{i}=1 \\
X_{i}>0
\end{array}\right.
\end{aligned}
$$




$$
\begin{gathered}
f(X)=\sum_{i=1}^{d} r_{i} x_{i}-\sum_{i=1}^{n}\left[\mu \cdot k_{i}^{b} \cdot\left(x_{i}-x_{i}^{0}\right)+(1-\mu) \cdot k_{i}^{s} \cdot\left(x_{i}^{0}-x_{i}\right)\right] \\
\text { and } \mu=\left\{\begin{array}{l}
1 X_{i} \geq X_{i}^{0} \\
0 X_{i}<X_{i}^{0}
\end{array}\right. \\
g(X)=\sum_{i=1}^{d} \sum_{j=1}^{d} X_{i} X_{j} \sigma_{i j} \\
h(X)=\sum_{i=1}^{d} \sum_{j=1}^{d} \sum_{k=1}^{d} x_{i} x_{j} x_{k} s_{i j k}
\end{gathered}
$$

Assuming that an investor will invest in $d$ assets. $\lambda$ represents the risk aversion factor. $f(X)$ represents investors' return function, $g(X)$ denotes risk function and $h(X)$ is skewness function. Let $X_{i}$ be the proportion of investment of asset $i, X_{i}^{0}$ be the initial holding proportion of investment, $r_{i}$ be the expected yields of $i$ th asset, $\sigma_{i j}$ be the covariance of $r_{i}$ and $r_{j}, s_{i j k}$ be the possibilistic co-skewness among $r_{i}, r_{j}$ and $r_{k} . k_{i}^{s}$ and $k_{i}^{b}$ denote the transaction fee for selling and buying the asset $i$ respectively.

\subsection{Encoding}

Encode the potentially feasible solution as ideas that individual generated in BFO-SDC. Four types of information including the value of corresponding profit, risk, and skewness, the proportion of investment are carried by each bacterium [25].

$$
\begin{gathered}
\theta=\left[X_{1}, X_{2}, X_{3} \ldots X_{d}, f(X), g(X), h(X)\right] \\
s=\sum_{i=1}^{d} X_{i} \\
\theta^{\prime}=\left[\frac{1}{s}\left(X_{1}, X_{2}, X_{3} \ldots X_{d}\right), f(X)^{\prime}, g(X)^{\prime}, h(X)^{\prime}\right]
\end{gathered}
$$

Equation (19) introduces the coding of the bacteria. As is illustrated in Eq. (20), all the assets' proportions are summed up at the beginning and then each proportion of asset is divided by the $s$ for the purpose of assuring all asset proportions' sum is equal to 1 .

\subsection{Constrained Boundary Control}

$$
\theta^{i}(j+1, k, l)= \begin{cases}D_{\min }+\text { rand } \cdot\left(D_{\max }-D_{\min }\right), & \text { if } P<0 \\ \theta^{i}(j, k, l)+C(i) \frac{\Delta(i)}{\sqrt{\Delta^{T}(i) \Delta(i)}}, & \text { if } P \geq 0\end{cases}
$$

Boundary control assurances that every proportion is positive. Bacteria may exceed the given area since in the process of chemotaxis they change directions randomly. The rules of boundary control are shown in Eq. (21), $D_{\max }$ and $D_{\min }$ are the upper and lower boundaries. And rand is a function that is used to generate a real number between 0 and 1 . 


\section{Experiments and Discussions}

For the purpose of testing the performance of the BFO-SDC algorithm, the original BFO and BFO-LDC are selected to compare. All the algorithms mentioned in this paper were coded in MATLAB R2018b.

\subsection{Definition of Experiments}

In the experiments, we assume that there are five assets can be selected to invest. Due to we suppose that the assets' future returns are trapezoidal fuzzy numbers, it's necessary to estimate the core interval, the right and left width of the fuzzy numbers. According to Vercher et al. [26], regard the historical returns as samples and use the sample percentile to approximate the spreads and core of the trapezoidal fuzzy returns. Thus, the interval of the 40th to 60th percentile is set as the core of the fuzzy return, the interval of the 5th to 40th percentile is set as the left-width and the interval of 40th to 95th percentile is set as the right-width. We consider the monthly returns over the tested period between January 2015 and December 2019. The trapezoidal fuzzy numbers are shown in Table 1.

Table 1. The trapezoidal fuzzy numbers

\begin{tabular}{l|l|l|l|l|l}
\hline The asset $i$ & 1 & 2 & 3 & 4 & \multicolumn{1}{l}{5} \\
\hline$\alpha$ & -0.10516 & -0.13309 & -0.30344 & -0.14459 & -0.08656 \\
\hline$a$ & -0.00244 & -0.01443 & 0.013429 & 0.004195 & 0.020846 \\
\hline$b$ & 0.042983 & 0.026308 & 0.06576 & 0.045895 & 0.05327 \\
\hline$\beta$ & 0.220344 & 0.160453 & 0.543263 & 0.254134 & 0.169063 \\
\hline
\end{tabular}

In this paper, we assume that investors are rational. Thus, the lower possibility variance is used to describe the risk. According to Theorem 1 and Definition 2, the risk of investment is shown as follows.

$$
\begin{gathered}
\operatorname{Var}^{-}\left(\sum_{i=1}^{d} x_{i} r_{i}\right)=\sum_{i=1}^{d} x_{i}^{2} \operatorname{Var}^{-}\left(r_{i}\right)+2 \sum_{i<j=1}^{d} x_{i} x_{j} \operatorname{Cov}^{-}\left(r_{i}, r_{j}\right) \\
=\left[\sum_{i=1}^{d} x_{i}\left(\frac{a_{i}-b_{i}}{2}+\frac{\alpha_{i}+\beta_{i}}{6}\right)\right]^{2}+\frac{1}{18}\left(\sum_{i=1}^{d} x_{i} \alpha_{i}\right)^{2}+2 \sum_{i<j=1}^{d} x_{i} x_{j} \operatorname{Cov}^{-}\left(r_{i}, r_{j}\right) \\
\operatorname{Cov}^{-}\left(r_{i}, r_{j}\right)=\left(\frac{b_{i}-a_{i}}{2}+\frac{\beta_{i}-a_{i}}{6}\right)\left(\frac{b_{j}-a_{j}}{2}+\frac{\beta_{j}-a_{j}}{6}\right)+\frac{\alpha_{j}}{3}\left(\frac{b_{i}-a_{i}}{2}+\frac{\beta_{i}-\alpha_{i}}{6}\right) \\
+\frac{\alpha_{i}}{3}\left(\frac{b_{j}-a_{j}}{2}+\frac{\beta_{j}-\alpha_{j}}{6}\right)+\frac{\alpha_{i} \alpha_{j}}{6}
\end{gathered}
$$


According to Liu et al. [27], the possibilistic skewness of the portfolio is

$$
\begin{aligned}
& S\left(\sum_{i=1}^{d} x_{i} r_{i}\right)=\frac{19}{1089}\left[\left(\sum_{i=1}^{d} x_{i} \beta_{i}\right)^{3}-\left(\sum_{i=1}^{d} x_{i} \alpha_{i}\right)^{3}\right] \\
& +\frac{1}{72}\left[\left(\sum_{i=1}^{d} x_{i} \alpha_{i}\right)\left(\sum_{i=1}^{d} x_{i} \beta_{i}\right)^{2}-\left(\sum_{i=1}^{d} x_{i} \beta_{i}\right)\left(\sum_{i=1}^{d} x_{i} \alpha_{i}\right)^{2}\right] \\
& +\frac{1}{24} \sum_{i=1}^{d} x_{i}\left(b_{i}-a_{i}\right)\left[\left(\sum_{i=1}^{d} x_{i} \beta_{i}\right)^{2}-\left(\sum_{i=1}^{d} x_{i} \alpha_{i}\right)^{2}\right]
\end{aligned}
$$

In the decreasing chemotaxis step length based on sine function, $C_{\min }=0.01, C_{\max }=$ 0.5 . The following parameter settings are the same as in corresponding reference [28]. To show various investors based on different preference to evaluate the final fortune, $\lambda$ is set as $0.15,0.5$ and 0.85 respectively. $k_{i}^{s}=0.00075$ and $k_{i}^{b}=0.00065$. In BFO, $N_{c}=1000$, $N_{e d}=2, N_{r e}=5, N_{s}$ (the number of swimming) $=4, P_{e d}$ (the elimination-dispersal frequency $)=0.25$. $C$ (the swimming length $)=0.2$.

\subsection{Experimental Results}

Numerical results with different $\lambda$ and the terminal portfolio selection results obtained by the original BFO, BFO-LDC, and BFO-SDC are showed in Tables 2, 3 and 4. Figure 1 shows the convergence curves with various $\lambda$ generated by the three algorithms.

Table 2. Experimental results of $\lambda=0.15$

\begin{tabular}{l|l|l|l}
\hline & BFO & BFO-LDC & BFO-SDC \\
\hline Min & $-2.9076 \mathrm{E}+00$ & $-1.7248 \mathrm{E}+01$ & $\mathbf{- 2 . 1 8 6 6 E + 0 1}$ \\
\hline Max & 0 & 1 & 0 \\
\hline Mean & $-2.9076 \mathrm{E}-01$ & $-1.7248 \mathrm{E}+00$ & $\mathbf{- 2 . 1 8 6 6 E + 0 0}$ \\
\hline Std. & $\mathbf{9 . 1 9 4 6 E}-01$ & $5.4544 \mathrm{E}+00$ & $6.9147 \mathrm{E}+00$ \\
\hline $\mathrm{X}_{1}$ & $2.3619 \mathrm{E}-01$ & $1.6276 \mathrm{E}-01$ & $1.8054 \mathrm{E}-01$ \\
\hline $\mathrm{X}_{2}$ & $1.6315 \mathrm{E}-01$ & $2.5461 \mathrm{E}-01$ & $2.0436 \mathrm{E}-01$ \\
\hline $\mathrm{X}_{3}$ & $2.7044 \mathrm{E}-01$ & $2.7919 \mathrm{E}-01$ & $1.4639 \mathrm{E}-01$ \\
\hline $\mathrm{X}_{4}$ & $1.3663 \mathrm{E}-01$ & $1.4928 \mathrm{E}-01$ & $2.6812 \mathrm{E}-01$ \\
\hline $\mathrm{X}_{5}$ & $1.9360 \mathrm{E}-01$ & $1.5416 \mathrm{E}-01$ & $2.0059 \mathrm{E}-01$ \\
\hline Return & $1.0320 \mathrm{E}-01$ & $1.0230 \mathrm{E}-01$ & $9.1529 \mathrm{E}-02$ \\
\hline Risk & $3.3634 \mathrm{E}-03$ & $3.3894 \mathrm{E}-03$ & $2.7046 \mathrm{E}-03$ \\
\hline Skewness & $3.1849 \mathrm{E}-04$ & $3.0871 \mathrm{E}-04$ & $2.1187 \mathrm{E}-04$ \\
\hline
\end{tabular}


Table 3. Experimental results of $\lambda=0.5$

\begin{tabular}{l|l|l|l}
\hline & BFO & BFO-LDC & BFO-SDC \\
\hline Min & $-1.2345 \mathrm{E}+00$ & $-3.0142 \mathrm{E}+00$ & $\mathbf{- 6 . 2 2 8 7 E + 0 0}$ \\
\hline Max & 0 & 0 & 0 \\
\hline Mean & $-1.2345 \mathrm{E}-01$ & $-3.0143 \mathrm{E}-01$ & $\mathbf{- 6 . 2 2 8 7 E}-\mathbf{0 1}$ \\
\hline Std. & $\mathbf{3 . 9 0 3 9 E}-\mathbf{0 1}$ & $9.5319 \mathrm{E}-01$ & $1.9697 \mathrm{E}+00$ \\
\hline $\mathrm{X}_{1}$ & $3.2366 \mathrm{E}-01$ & $2.4145 \mathrm{E}-01$ & $2.7598 \mathrm{E}-01$ \\
\hline $\mathrm{X}_{2}$ & $9.0457 \mathrm{E}-02$ & $6.1790 \mathrm{E}-02$ & $2.3570 \mathrm{E}-01$ \\
\hline $\mathrm{X}_{3}$ & $1.8598 \mathrm{E}-01$ & $2.9873 \mathrm{E}-01$ & $9.1497 \mathrm{E}-02$ \\
\hline $\mathrm{X}_{4}$ & $2.0407 \mathrm{E}-01$ & $8.8298 \mathrm{E}-02$ & $1.0163 \mathrm{E}-01$ \\
\hline $\mathrm{X}_{5}$ & $1.9583 \mathrm{E}-01$ & $3.0973 \mathrm{E}-01$ & $2.9520 \mathrm{E}-01$ \\
\hline Return & $9.6784 \mathrm{E}-02$ & $1.0777 \mathrm{E}-01$ & $8.2556 \mathrm{E}-02$ \\
\hline Risk & $2.9941 \mathrm{E}-03$ & $3.4405 \mathrm{E}-03$ & $2.2346 \mathrm{E}-03$ \\
\hline Skewness & $2.6747 \mathrm{E}-04$ & $3.4922 \mathrm{E}-04$ & $1.5155 \mathrm{E}-04$ \\
\hline & & &
\end{tabular}

Table 4. Experimental results of $\lambda=0.85$

\begin{tabular}{l|l|l|l}
\hline & BFO & BFO-LDC & BFO-SDC \\
\hline Min & $-2.5908 \mathrm{E}-02$ & $-2.6722 \mathrm{E}-02$ & $\mathbf{- 2 . 7 7 8 5 E}-\mathbf{0 2}$ \\
\hline Max & 0 & 0 & 0 \\
\hline Mean & $-2.5908 \mathrm{E}-03$ & $-2.6722 \mathrm{E}-03$ & $\mathbf{- 2 . 7 7 8 5 E}-\mathbf{0 3}$ \\
\hline Std. & $\mathbf{8 . 1 9 2 8 E}-\mathbf{0 3}$ & $8.4501 \mathrm{E}-03$ & $8.7863 \mathrm{E}-03$ \\
\hline $\mathrm{X}_{1}$ & $8.4933 \mathrm{E}-02$ & $1.4236 \mathrm{E}-01$ & $1.5807 \mathrm{E}-01$ \\
\hline $\mathrm{X}_{2}$ & $2.0848 \mathrm{E}-01$ & $2.4435 \mathrm{E}-01$ & $2.1004 \mathrm{E}-01$ \\
\hline $\mathrm{X}_{3}$ & $3.0795 \mathrm{E}-01$ & $2.1466 \mathrm{E}-01$ & $3.2087 \mathrm{E}-01$ \\
\hline $\mathrm{X}_{4}$ & $2.0448 \mathrm{E}-01$ & $2.5917 \mathrm{E}-01$ & $9.4012 \mathrm{E}-02$ \\
\hline $\mathrm{X}_{5}$ & $1.9415 \mathrm{E}-01$ & $1.3945 \mathrm{E}-01$ & $2.1701 \mathrm{E}-01$ \\
\hline Return & $1.0742 \mathrm{E}-01$ & $9.7463 \mathrm{E}-02$ & $1.0697 \mathrm{E}-01$ \\
\hline Risk & $3.5742 \mathrm{E}-03$ & $3.1070 \mathrm{E}-03$ & $3.5597 \mathrm{E}-03$ \\
\hline Skewness & $3.4445 \mathrm{E}-04$ & $2.6401 \mathrm{E}-04$ & $3.4545 \mathrm{E}-04$ \\
\hline & & &
\end{tabular}



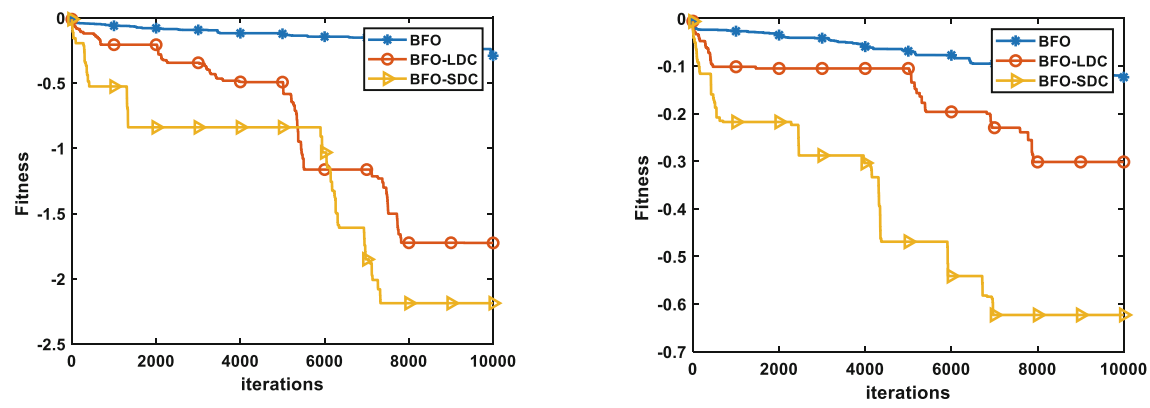

$\lambda=0.15$

$\lambda=0.5$

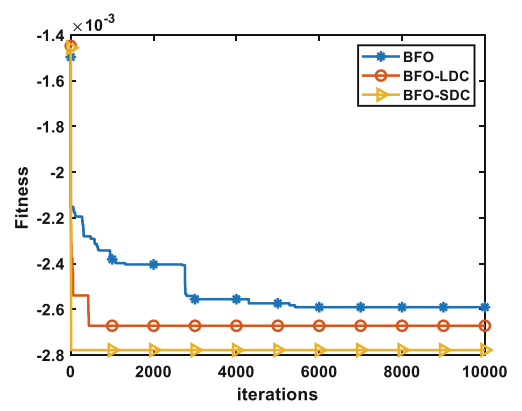

$\lambda=0.85$

Fig. 1. The convergence curve of three algorithms

According to the tables and the figures, we can find that:

(1) The fitness value grows up with the increase of the risk-averse factor $\lambda$, and this trend keeps the same with the structure of the fitness function.

(2) With the different $\lambda$ and different algorithm, the percentage of five assets is not the same. The results show that lower risk can be with the less profit.

(3) Comparing the numerical results shown in Tables 2 and 3, it verifies that BFO-SDC outperforms BFO and BFO-LDC in terms of mean value, minimum value and result qualify, which clarifies the effectiveness of the BFO-SDC.

(4) Comparing the convergence graphs shown in Fig. 1, BFO-SDC is superior to BFO$\mathrm{LDC}$ and $\mathrm{BFO}$ for the three cases.

\section{Conclusions and Future Work}

In this paper, we pay attention to solving the PO problem by using BFO based methods. Based upon the standard mean-variance model, we take skewness and fuzzy set theory into consideration and use semivariance to take the place of variance. A decreasing chemotaxis step strategy based on sine function is included in the original BFO to solve 
this model. The obtained results indicate that BFO-SDC outperforms than BFO and BFO-LDC.

Further works may consider some biological mechanisms to promote the performance of BFO and propose novel models containing other conditions such as dynamic objectives or multi-period to meet the demands from the real-world.

Acknowledgements. This work is partially supported by Shenzhen Philosophy and Social Sciences Plan Project (SZ2019D018), Guangdong Provincial Soft Science Project (2019A101002075).

\section{References}

1. Markowitz, H.M.: Portfolio selection. J. Financ. 7, 77-91 (1952)

2. Zhou, Z., Ren, T., Xiao, H., Liu, W.: Time-consistent investment and reinsurance strategies for insurers under multi-period mean-variance formulation with generalized correlated returns. J. Manag. Sci. Eng. 4, 142-157 (2019)

3. Bi, J., Jin, H., Meng, Q.: Behavioral mean-variance portfolio selection. Eur. J. Oper. Res. 271, 644-663 (2018)

4. Chunhachinda, P., Dandapani, K., Hamid, S., Prakash, A.J.: Portfolio selection and skewness: evidence from international stock markets. J. Bank. Financ. 21, 143-167 (1997)

5. Simkowitz, M.A., Beedles, W.L.: Diversification in a three-moment world. J. Financ. Quant. Anal. 13, 927-941 (1978)

6. Ibbotson, R.G.: Price performance of common stock new issues. J. Financ. Econ. 2, 235-272 (1975)

7. Prakash, A.J., Chang, C.H., Pactwa, T.E.: Selecting a portfolio with skewness: recent evidence from US, European, and Latin American equity markets. J. Bank. Financ. 27, 1375-1390 (2003)

8. Markowitz, H.: Portfolio Selection: Efficient Diversification of Investments. Wiley, New York (1959)

9. Ammar, E., Khalifa, H.A.: Fuzzy portfolio optimization a quadratic programming approach. Chaos, Solitons Fractals 18, 1045-1054 (2003)

10. Liu, Y., Passino, K.M.: Biomimicry of social foraging bacteria for distributed optimization: models, principles, and emergent behaviors. J. Optim. Theory Appl. 115, 603-628 (2002)

11. Niu, B., Tan, L.J., Liu, J., Liu, J., Yi, W.J., Wang, H.: Cooperative bacterial foraging optimization method for multi-objective multi-echelon supply chain optimization problem. Swarm Evol. Comput. 49, 87-101 (2019)

12. Vital-Soto, A., Azab, A., Baki, M.F.: Mathematical modeling and a hybridized bacterial foraging optimization algorithm for the flexible job-shop scheduling problem with sequencing flexibility. J. Manuf. Syst. 54, 74-93 (2020)

13. Tan, L., Lin, F., Wang, H.: Adaptive comprehensive learning bacterial foraging optimization and its application on vehicle routing problem with time windows. Neurocomputing 151, 1208-1215 (2015)

14. Niu, B., Fan, Y., Xiao, H., Xue, B.: Bacterial foraging based approaches to portfolio optimization with liquidity risk. Neurocomputing 98, 90-100 (2012)

15. Tang, W.K., Zhong, G., Chen, G., Man, K.F.: Generation of N-scroll attractors via sine function. IEEE Trans. Circuits Syst. I: Regular Pap. 48, 1369-1372 (2001)

16. Zhang, X., Zhang, G.: Design of ship course-keeping autopilot using a sine function-based nonlinear feedback technique. J. Navig. 69, 246-256 (2016) 
17. Volos, C., Akgul, A., Pham, V., Stouboulos, I., Kyprianidis, I.: A simple chaotic circuit with a hyperbolic sine function and its use in a sound encryption scheme. Nonlinear Dyn. 89, 1047-1061 (2017)

18. Zadeh, L.A.: Fuzzy sets as a basis for a theory of possibility. Fuzzy Sets Syst. 1, 3-28 (1978)

19. Zadeh, L.A.: Fuzzy sets. Inf. Control 8, 338-353 (1965)

20. Carlsson, C., Fuller, R.: On possibilistic mean value and variance of fuzzy numbers. Fuzzy Sets Syst. 122(2), 315-326 (2001)

21. Saeidifar, A., Pasha, E.: The possibilistic moments of fuzzy numbers and their applications. J. Comput. Appl. Math. 2, 1028-1042 (2009)

22. Zhang, W.G., Liu, Y.J., Xu, W.J.: A possibilistic mean-semivariance-entropy model for multiperiod portfolio selection with transaction costs. Eur. J. Oper. Res. 222, 341-349 (2012)

23. Zhang, W.G., Wang, Y.L., Chen, Z.P., Nie, Z.K.: Possibilistic mean-variance models and efficient frontiers for portfolio selection problem. J. Inf. Sci. 177, 2787-2801 (2007)

24. Li, X., Qin, Z., Kar, S.: Mean-variance-skewness model for portfolio selection with fuzzy returns. Eur. J. Oper. Res. 202, 239-247 (2010)

25. Niu, B., Bi, Y., Xie, T.: Structure-redesign-based bacterial foraging optimization for portfolio selection. In: Huang, D.-S., Han, K., Gromiha, M. (eds.) ICIC 2014. LNCS, vol. 8590, pp. 424 430. Springer, Cham (2014). https://doi.org/10.1007/978-3-319-09330-7_49

26. Vercher, E., Bermudez, J., Segura, J.: Fuzzy portfolio optimization under downside risk measures. J. Fuzzy Sets Syst. 158, 769-782 (2007)

27. Liu, Y.J., Zhang, W.G., Zhao, X.J.: Fuzzy multi-period portfolio selection model with discounted transaction costs. Soft. Comput. 22, 177-193 (2018)

28. Niu, B., Liu, J., Liu, J., Yang, C.: Brain storm optimization for portfolio optimization. In: Tan, Y., Shi, Y., Li, L. (eds.) ICSI 2016. LNCS, vol. 9713, pp. 416-423. Springer, Cham (2016). https://doi.org/10.1007/978-3-319-41009-8_45 